\title{
Entanglement in Qutrit Systems
}

\author{
J. D. Huerta-Morales \\ Instituto Nacional de Astrofísica, Óptica y Electrónica (INAOE), Puebla, Mexico \\ del_hm5@hotmail.com
}

\begin{abstract}
Wootters concurrence is an entanglement measure for bipartite qubit systems. It is defined in terms of a spin inverter. Here, we try to generalize Wootters concurrence for qutrit systems by defining a naïve inverter analogous to the $S U(2)$ qubit inverter given in terms of Pauli matrix $\sigma_{y}$. For qutrits, the corresponding group is $S U(3)$, so the inverter has to be given in terms of GellMann matrices. The naïve inverter proposed here is given in terms of the GellMann matrices analogous to $\sigma_{y}$ and we show that it can deliver equivalent information to that given by the formal inverter in just in certain cases.
\end{abstract}

Keywords: Entanglement, qutrit system.

\section{Introduction}

Quantum entanglement is a precious resource, thus, there is a need to measure how entangled a quantum systems. Various entanglement measures have been proposed in the literature, but Wootters concurrence is probably the most widely used.

A measure of entanglement in a bipartite qubit system is Wootters concurrence. It is defined in terms of a superoperator that rotates the qutrit spin [1-3]. In this work, we pretend to construct a naïve bipartite qutrit inverter based on Gell-Mann matrices which may be considered as analogous to the superoperator represented by Pauli matrices but, as we will show, is not a proper universal inverter.

Wootters concurrence of a pure state for a qutrit system is defined as follows,

$$
C_{3}(\Psi) \equiv \sqrt{\operatorname{tr}(\rho \tilde{\rho})},
$$

where $\rho$ is the density matrix and $\tilde{\rho}$ is given in terms of the naïve inverter,

$$
\tilde{\rho}=S_{3} \otimes S_{3}(\rho)=S_{y} \otimes S_{y} \rho * S_{y} \otimes S_{y} .
$$

In this work we study entanglement in a qutrit beam splitter and compare the results produced by the formal inverter and the naïve construction,

$$
S_{3} \otimes S_{3}(\rho)=V_{3} V_{3}\left[I \otimes I-I \otimes \rho_{B}-\rho_{A} \otimes I+\rho\right],
$$

where $I$ is the unitary matrix. 


\section{$2 \quad$ Naïve Inverter for a Qutrit System}

Let us consider a bipartite qutrit system; a general state vector is given by,

$$
|\Psi\rangle_{A B}=\sum_{i, j=0}^{2} C_{i j}|\mathrm{i}\rangle_{A} \otimes|\mathrm{j}\rangle_{B},
$$

where the Hilbert space for each qutrit system is span by the vector basis,

$$
|2\rangle=\left(\begin{array}{l}
1 \\
0 \\
0
\end{array}\right),|1\rangle=\left(\begin{array}{l}
0 \\
1 \\
0
\end{array}\right) \text { and }|0\rangle=\left(\begin{array}{l}
0 \\
0 \\
1
\end{array}\right) .
$$

We will consider only pure state $\rho=|\Psi\rangle_{A B}\langle\Psi|$, and propose a naïve inverter given in terms of a $S_{3}$ superoperator,

$$
S_{3}(\rho)=S_{y} \rho^{*} S_{y},
$$

where the auxiliary matrix is,

$$
S_{y}=\lambda_{2}+\lambda_{5}+\lambda_{7}=\left(\begin{array}{ccc}
0 & -i & -i \\
i & 0 & -i \\
i & i & 0
\end{array}\right),
$$

is choose as an analogous to Pauli matrix $\sigma_{y}$. Of course, this naïve inverter is not the formal inverter corresponding $S U(3)$ but we will choose that it can provide equivalent results in some specific cases.

It is straightforward to calculate Wootters concurrence for the formal inverter,

$$
\begin{aligned}
C_{f}(\Psi) \equiv \sqrt{\operatorname{tr}(\rho \tilde{\rho})} & =\sqrt{\sum\langle\Psi \mid \Psi\rangle\left\langle\Psi\left|S_{3} \otimes S_{3}(|\Psi\rangle\langle\Psi|)\right| \Psi\right\rangle} \\
& =\sqrt{\left\langle\Psi\left|S_{3} \otimes S_{3}(|\Psi\rangle\langle\Psi|)\right| \Psi\right\rangle},
\end{aligned}
$$

and the naïve inverter,

$$
\begin{aligned}
C_{n}(\Psi)=\sqrt{\sum\langle\Psi \mid \Psi\rangle\left\langle\Psi\left|S_{y} \otimes S_{y}\left(\left|\Psi^{*}\right\rangle\left\langle\Psi^{*}\right|\right) S_{y} \otimes S_{y}\right| \Psi\right\rangle} \\
=\sqrt{\left\langle\Psi\left|S_{y} \otimes S_{y}\right| \Psi^{*}\right\rangle\left\langle\Psi\left|S_{y} \otimes S_{y}\right| \Psi^{*}\right\rangle^{*}} \\
=\left|\left\langle\Psi\left|S_{y} \otimes S_{y}\right| \Psi^{*}\right\rangle\right| .
\end{aligned}
$$

\section{Results}

We want to test the results provided by the naïve inverter, which requires less computer resources to calculate, versus those given by the formal inverter. First, we will study a simple test state and, then, we will use as test state the time evolution of two different states under beam splitter dynamics. 


\subsection{A simple Test State}

The first test sate is given by the following expression,

$$
|\Psi\rangle=\sin \theta|20\rangle+\cos \theta|02\rangle .
$$

It becomes a separable state for $\theta=0$ and entangled for all other cases up to a maximum reached at $\theta=\pi / 4$. In this case, the density matrix is,

$$
\rho=\sin ^{2} \theta|20\rangle\langle 20|+\sin \theta \cos \theta| 20\rangle\langle 02|+\cos \theta \sin \theta| 02\rangle\left\langle 20\left|+\cos ^{2} \theta\right| 02\right\rangle\langle 02| .
$$

We can calculate Wootters concurrence for both the formal and naïve inverter, which are presented in figure 1 where we can see that the formal and naïve inverter, provide the same information in this case.

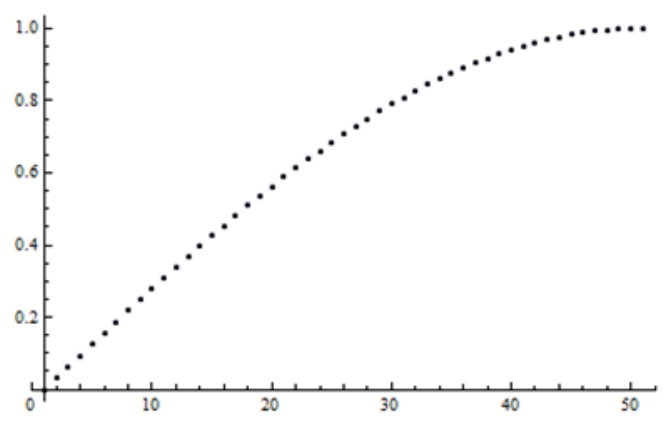

Fig. 1 Wootters concurrence from formal and naive inverter for the test state. The results are identical.

\subsection{Beam Splitter}

Propagation of two quantized electromagnetic fields through a beam splitter is given the time evolution operator,

$$
U(t)=e^{-i\left(a_{1}^{\uparrow} a_{2}+a_{2}^{\uparrow} a_{1}\right) t}
$$

Such that at any given time the state of the two-qutrits are given by,

$$
|\Psi(t)\rangle=U(T)|\Psi(0)\rangle
$$

We are interested in the evolution of an initial entangled state,

$$
\text { a) }|\Psi(0)\rangle=\frac{1}{\sqrt{2}}(|20\rangle+|02\rangle) \text {, }
$$

that yields a time propagated state vector, 


$$
\begin{gathered}
|\Psi(t)\rangle=\frac{1}{\sqrt{2}}\left(\cos ^{2} \theta(t)-\sin ^{2}(t)\right)|20\rangle-i 2 \cos (t) \sin (t)|11\rangle \\
+\frac{1}{\sqrt{2}}\left(\cos ^{2} \theta(t)-\sin ^{2}(t)\right)|02\rangle .
\end{gathered}
$$

The figure 2 shows Wootters concurrences calculated via the formal and naïve inverters, it is possible to see that both measures provide qualitatively the same information.

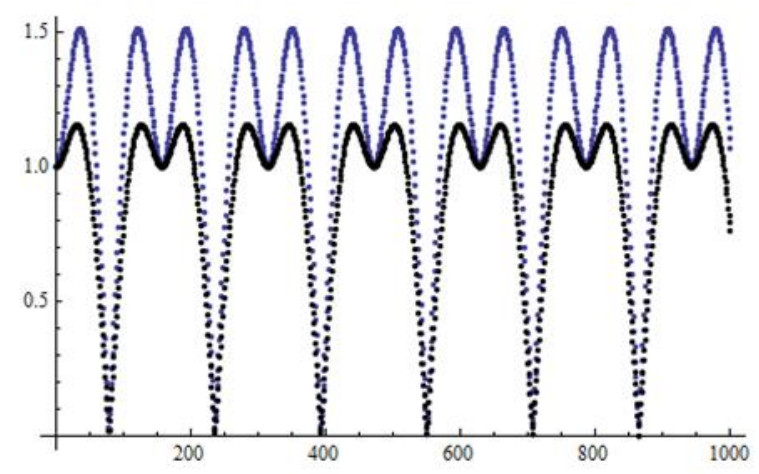

Fig. 2 Wootters concurrence from formal (black dots) and naïve inverter (blue dots) for the test entangled state Eq. (14).

We are also interested in the evolution of an initial separable state,

$$
\text { b) }|\Psi(0)\rangle=|20\rangle \text {, }
$$

which yields,

$$
|\Psi(t)\rangle=\cos ^{2}(t)|20\rangle-i \sqrt{2} \cos (t) \sin (t)|11\rangle-\sin ^{2}(t)|20\rangle .
$$

After calculating the density matrix, we get partial matrices, which are,

and,

$$
\rho_{A}(t)=\cos ^{4}(t)|2\rangle\left\langle 2\left|+2 \cos ^{2}(t) \sin ^{2}(t)\right| 1\right\rangle\left\langle 1\left|+\sin ^{4}(t)\right| 0\right\rangle\langle 0|,
$$

$$
\rho_{B}(t)=\sin ^{4}(t)|2\rangle\left\langle 2\left|+2 \cos ^{2}(t) \sin ^{2}(t)\right| 1\right\rangle\left\langle 1\left|+\cos ^{4}(t)\right| 0\right\rangle\langle 0| .
$$

Finally, the Wootters concurrences are:

$$
C_{f}=\frac{1}{8}(13+3 \cos (4 t)) \sin ^{2}(2 t),
$$

and

$$
C_{n}=\frac{1}{8}(9+7 \cos (4 t)) \sin ^{2}(2 t)
$$


that are shown in figure 3. It is straightforward to notice that the naïve inverter does not provide the same information than the formal inverter in this case.

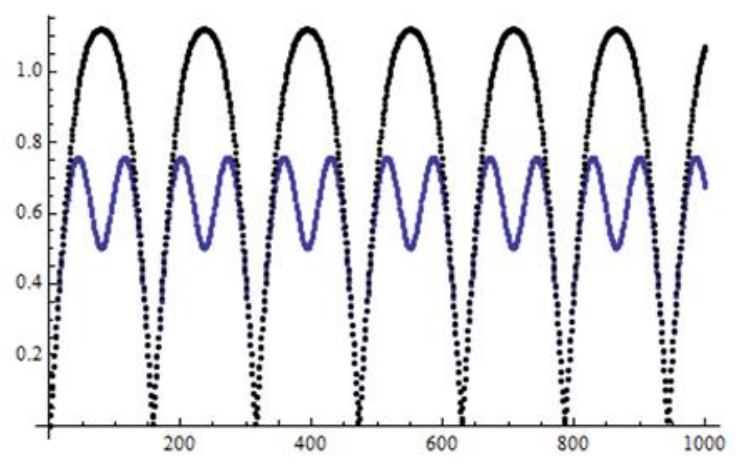

Fig. 3 Wootters the test state in Eq. (16).concurrence from formal (black dots) and naive inverters (blue dots) for the test state in Eq. (16).

\section{Conclusions}

We have proposed a naïve inverter for bipartite qutrit systems that reduces the computation time used to calculate Wootters concurrence for pure states. This naïve inverter is given in terms of Gell-Mann matrices but it is not a universal inverter. We have shown that in certain cases it provides results that are qualitative equal to those given by the formal inverter but it is not viable for any general state. We will continue exploring entanglement in bipartite qutrit systems through the use of naïve inverters for both pure and mixed states in the future.

Acknowledgments. The author is grateful to his master thesis supervisors, Dr. H. M. Moya Cessa and Dr. B. M. Rodriguez Lara, for their support.

\section{References}

1. Rungta, P., Buzek, V., Caves, C. M., Hillery, M., Milburn, G. J.: Phys. Rev. 64, 042315 (2001)

2. Hill, S., Wootters, W. K.: Phys. Rev. Lett. 78, 5022 (1997)

3. Wootters, W. K.: Phys. Rev. Lett. 80, 2245 (1998) 